\title{
Editorial:
}

\section{Host Related Genetic and Nutrition Factors Play the Key Roles in COVID-19 Severity}

Tooba Ghazanfari ${ }^{1^{*}}$ (D)

1. Immunoregulation Research Center, Shahed University, Tehran, Iran.

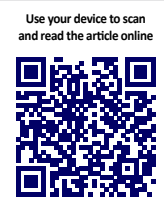

Citation Ghazanfari T. Host Related Genetic and Nutrition Factors Play the Key Roles in COVID-19 Severity. Immunoregulation. 2022; 4(2):67-68. http://dx.doi.org/10.32598/Immunoregulation.4.2.8

dol http://dx.doi.org/10.32598/Immunoregulation.4.2.8

$\mathrm{n}$ the editorial of this journal's two previous consecutive issues, it was noted that the I Coronavirus Disease 2019 (COVID-19) susceptibility and severity are strongly related to individual and familial factors. Thus, genetics and nutrition are essential among these host-related factors.

Numerous studies have been conducted in Caucasian populations that examined the genetic factors in $\mathrm{CO}$ VID-19, which Valavan et al. has well reviewed [1]. In their review, host-related genetic factors have been studied in different ways, including Genome-Wide Association Studies (GWAS), Whole-Exome Sequencing (WES), candidate gene studies, and essential genes involved in disease severity were introduced [1].

However, it is necessary to perform genomic studies, especially gene polymorphisms in different populations. As in our national survey at the Immunoregulation Research Center entitled: Iran-Cohort Study of COVID-19 Immunology and Immunopathology, differences in some polymorphisms were observed with other studies on the different populations.

Furthermore, despite its great importance as one of the most significant host factors that effectively prevents dis- ease, its emphasis on extensive studies, and its impact on the immune responses, nutrition has received less attention in COVID-19. The results of our cohort study indicated that using a healthy, not inflammatory, diet and plant-based diet throughout life is among the chief factors preventing the progression of COVID-19 severity.

The author emphasizes that genetic and nutritional studies in different populations and individuals could effectively identify the host response to the SARS-CoV-2 virus and prevent the severity of the COVID-19 disease.

\section{Ethical Considerations}

\section{Compliance with ethical guidelines}

There were no ethical considerations to be considered in this research.

Funding

This research did not receive any grant from funding agencies in the public, commercial, or non-profit sectors.

\section{Conflicts of interest}

The authors declared no conflict of interest.

\footnotetext{
* Corresponding Author:

Tooba Ghazanfari, PhD.

Address: Immunoregulation Research Center, Shahed University, Tehran, Iran.

Phone: +98 (21) 66418216

E-mail:tghazanfari@yahoo.com
} 


\section{References}

[1] Velavan TP, Pallerla SR, Rüter J, Augustin Y, Kremsner PG, Krishna S, et al. Host genetic factors determining COVID-19 susceptibility and severity. EBioMedicine. 2021; 72:103629. [DOI:10.1016/j.ebiom.2021.103629] [PMID] [PMCID] 